\title{
Interaction Between Gravity Compensation Suspension System and Deployable Structure
}

\author{
A. Fischer* and S. Pellegrino ${ }^{\dagger}$ \\ University of Cambridge, Cambridge, England CB2 1PZ, United Kingdom
}

\begin{abstract}
Gravity compensation suspension systems are essential to support space structures during tests on Earth, but also impose constraints on the structures that have the effect of changing their behavior. A computational and experimental study of the interaction of a rigid panel solar array model with a manually adjustable suspension system during quasi-static deployment tests in the 1- $g$ environment of the laboratory is presented. A methodology is established for modeling this interaction, for predicting the effects of suspension system adjustments, and for optimization of the suspension system through these adjustments. Some improvements can be achieved by manual adjustments, but further optimization requires an active system.
\end{abstract}

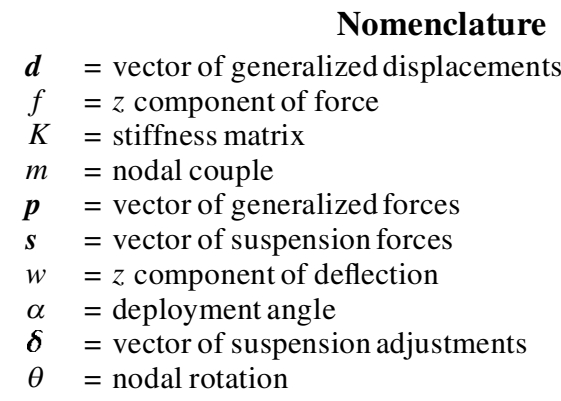

Subscripts

$A=$ array

$C=$ combined

$M=$ measured

$P \quad=$ predicted

$R=$ required

$S \quad=$ suspension

Superscript

$\sim \quad=$ full (i.e., uncondensed)

\section{Introduction}

$\mathbf{T}$ HE 0- $g$ environment of space makes it possible to design large space structures of low mass. The dimensions of such structural systems pose a problem during transportinto orbit, however, because the payload volume of launchers is limited. Therefore, a variety of deployable structures are used that can be packaged into a small volume and, once in space, can be deployed into their operating configuration.

Prior to flight, ground validationtests of such structures are carried out to ensure reliable and accurate performance in space. However, the 1- $g$ environment and the associated self-weight loading on the structure have to be counteracted with an artificial support system. The problem is that this system imposes constraints on the structure, and, thus, perturbs its static, dynamic, and deployment behavior.

Presented as Paper 98-1835 at the AIAA/ASME/ASCE/AHS/ASC 39th Structures, Structural Dynamics, and Materials Conference, Long Beach, CA, 20-23 April 1998; received 18 June 1998; revision received 16 May 1999; accepted for publication 27 June 1999. Copyright () 1999 by A. Fischer and S. Pellegrino. Published by the American Institute of Aeronautics and Astronautics, Inc., with permission.

${ }^{*}$ Research Student, Department of Engineering, Trumpington Street. Student Member AIAA.

${ }^{\dagger}$ Reader in Structural Engineering, Department of Engineering, Trumpington Street; pellegrino@eng.cam.ac.uk. Associate Fellow AIAA.
Gravity compensation systems play a key role in replicating as closely as possible the $0-g$ conditions of space. Concepts used for the testing of space structures include physical methods such as drop towers and parabolic flight maneuvres, ${ }^{1}$ buoyancy techniques, air bearings/tables, and simple mechanical suspension systems featuring cables and pulleys, often in combination with counterweights, zero-springrate mechanisms, and pneumatic/electric devices. ${ }^{2,3} \mathrm{Im}$ provement of the mechanical methods lead to the development of actively controlled single-point suspension systems. ${ }^{4-10}$

To support the large-scale deployment motion of modern space structures, passive and, more recently, actively controlled multipoint suspension systems are used. ${ }^{11,12}$ However, the inherent flexibility of deployable structures is the source of complex interactions between structure and suspension system that requires careful examination. To obtain reliable predictions from ground tests for the deployment behavior in space, these interactions have to be understood.

The particular deployable structure that is investigated in this paper is a cable-deployedrigid panel solar array of the type used in the European Retrievable Carrier (EURECA) spacecraft. This type of solar array exhibits features typical of large deployable space structures such as high, variable flexibility, and, hence, multiple supports are required to prevent excessive loading and deformation. Deployment tests on a small-scale laboratory mode ${ }^{13}$ have revealed a complex interaction with the support system; the variations in the suspension forces observed even during quasi-static deployment were surprisingly large. The aim of this paper is to developanalytical models to capture this interaction, and then to adjust the suspension system to isolate as much as possible the structural behavior of the test article from that of its suspension.

Following a brief description of the model structure that will be investigated, the section Computational Models sets up the configuration-dependent stiffness matrix of the array and the constant stiffness matrix of its suspension system. Thus, a relationship between the adjustments of the suspensionsystem and the associated changes in the suspensionforces, for any configuration of the array, is obtained. In the section Variation of Suspension Forces During Deployment, it is found that there are considerable differences between the predicted, the experimentally observed, and the required suspension forces. Thus, in the following section a simple method for adjusting the suspension system and achieving predictable behavior is established. It is concluded that the suspension system currently used, which does not allow on-line adjustment, cannot produce accurate gravity compensation, but even a simple, actively controlled system would be able to.

\section{Physical Model of Solar Array}

The solar array that is examined in this paper is a simplified version of the retractable advanced rigid array of the EURECA mission. Figure 1 is a schematic view of the test rig, including the solar array model and the suspension system. Geometrical and material properties are listed in Table 1 . 
Table 1 Properties of solar array model

\begin{tabular}{lc}
\hline \hline Parameter & \multicolumn{1}{c}{ Value } \\
\hline & Dimensions \\
Array & Fully deployed length $2,200 \mathrm{~mm}$ \\
Panels & $400(200) \times 100 \times 1.63 \mathrm{~mm}$ \\
& Panel material \\
Al-alloy & $E=70,000 \mathrm{~N} / \mathrm{mm}^{2}$ \\
& $v=0.33$ \\
& $\rho=0.0027 \mathrm{~g} / \mathrm{mm}^{3}$ \\
& Mass distribution \\
Total mass & $4,343 \mathrm{~g}$ \\
Tip mass & $355 \mathrm{~g}$ \\
Mass of panels & $0.405 \mathrm{~g} / \mathrm{mm}$ \\
Mass of hinges & $m_{1}=310 \mathrm{~g}$ \\
& $m_{2, \ldots, 6}=507 \mathrm{~g}$ \\
& $m_{7}=607 \mathrm{~g}$ \\
\hline \hline
\end{tabular}

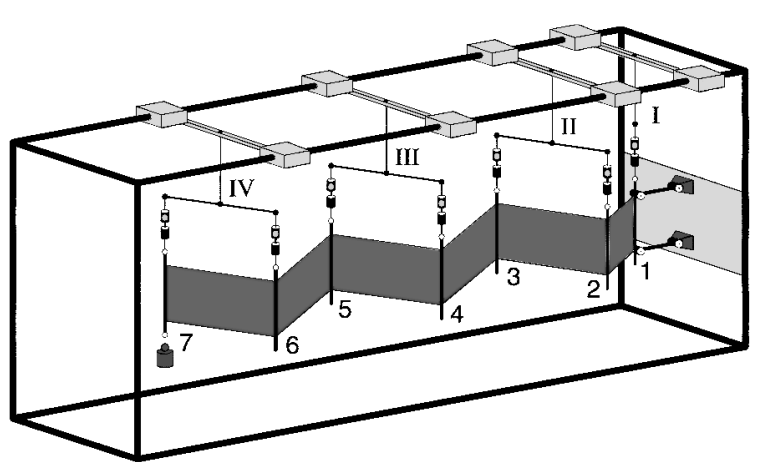

Fig. 1 Schematic of solar array model and suspension system.

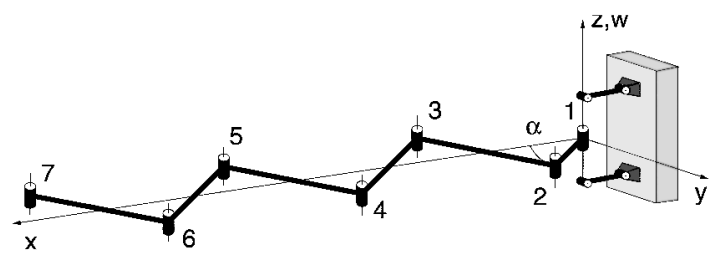

Fig. 2 Beam element model of array.

The model array structure consists of rectangular Al-alloy panels that are hinged to each other along the short vertical edges. There are five full-length panels, plus a half-length panel serving the purpose of a yoke, that separates the array structure from the spacecraft. The structure can move in a horizontal plane, concertinalike.Its deployment and retraction are driven by cables running over a pulley system and wound on a single motorized drum. The motion is synchronized by closed-contact-loop cables that link adjacent panels in such a way that they rotate by equal and opposite amounts. The deployment drum is driven by a stepper motor controlled via a personal computer. Further details on the design of this experiment are available elsewhere. ${ }^{13}$

Gravity compensation is achieved by means of three identical, movable double-point suspension elements. Each element consists of a horizontal tube connected by steel cables, terminated by steel rings, to the suspension points of the array. These tubes are suspended via steel shafts from Al-alloy rods supported on linear bearings that run on horizontal steel rails above the structure. The root of the array is held by a single suspension element and fixed to a thick base plate, such that only translation in the vertical direction is permitted. Altogether, there are seven suspension points, located above the hinge shafts. They counteract the gravity loading on the array, almost at the points where it arises, because most of the array mass is concentrated at the hinges.

Each suspension cable has a turnbuckle for length adjustment, mounted in series with a strain gauge to measure the cable tension. Data from these strain gauges are recorded with a data logger throughoutdeployment. Length adjustments are made manually and checked with a Vernier caliper before deployment. However, no adjustment can be made during deployment.
To avoid undesirable deformation of the array structure due to an unbalanced weight distribution amongst the suspension points, a tip mass is attached to the last hinge adding the weight of approximately half of a hinge and half of a panel.

\section{Computational Models}

To investigate the interaction between the array structure and its suspension system, finite element models are set up for each, relating the displacements of the suspension points to the applied forces. Then the individual models are combined, with and without consideration of the length changes in the suspension cables, to derive a computational model of the complete system. Thus, a relationship between the length adjustments and the redistribution of the suspension forces is obtained.

\section{Model of Solar Array}

The solar array is modeled as an assembly of six beam elements (Fig. 2) connected by revolute joints with vertical axes of rotation. Nodes 1-7 coincide with the hinges and with the suspension points. We are only interested in the vertical displacements at the suspension points and, hence, only in the out-of-plane stiffness of the array. According to an in-plane model of the array, ${ }^{13}$ the in-plane and out-of-plane behavior of the array are decoupled, provided that the out-of-plane deflections are small. The state of deployment or retraction is uniquely defined by the deploymentangle $\alpha$, with $\alpha=0$ and 90 deg denoting the fully deployed and fully retracted configurations, respectively.For our study, it makes no difference whether the array is being deployed or retracted, and, hence, only deployment will be mentioned from now on.

The stiffness matrix $\tilde{\boldsymbol{K}}_{A}$ of the array structure, relating the displacements $\tilde{\boldsymbol{d}}_{A}$ to the forces $\tilde{\boldsymbol{p}}_{A}$ at the suspension points, is composed of the standard stiffness matrices for the individual beams. Here, $\tilde{\boldsymbol{d}}_{A}$ includes the vertical displacement components of all of the nodes plus the rotations $\theta_{x}$ and $\theta_{y}$ of nodes 2-7. Therefore, the stiffness matrix $\tilde{\boldsymbol{K}}_{A}$ of the array has dimensions $(19 \times 19)$, and the forcedisplacement relationship is given by

$$
\tilde{\boldsymbol{K}}_{A} \tilde{\boldsymbol{d}}_{A}=\tilde{\boldsymbol{K}}_{A}\left[\begin{array}{c}
\boldsymbol{w}_{A} \\
\boldsymbol{\theta}_{x_{A}} \\
\boldsymbol{\theta}_{y_{A}}
\end{array}\right]=\left[\begin{array}{c}
\boldsymbol{f}_{A} \\
\boldsymbol{m}_{x_{A}} \\
\boldsymbol{m}_{y_{A}}
\end{array}\right]=\tilde{\boldsymbol{p}}_{A}
$$

or, in block form,

$$
\left(\begin{array}{cc}
\tilde{\boldsymbol{K}}_{A_{w w}} & \tilde{\boldsymbol{K}}_{A_{w \theta}} \\
\tilde{\boldsymbol{K}}_{A_{\theta w}} & \tilde{\boldsymbol{K}}_{A_{\theta \theta}}
\end{array}\right)\left(\begin{array}{c}
\boldsymbol{w}_{A} \\
\boldsymbol{\theta}_{A}
\end{array}\right)=\left(\begin{array}{c}
\boldsymbol{f}_{A} \\
\boldsymbol{m}_{A}
\end{array}\right)
$$

Because we are not interested in the rotation components, we use standard matrix condensation techniques to obtain

$$
\boldsymbol{K}_{A} \boldsymbol{w}_{A}=\boldsymbol{p}_{A}
$$

with

$$
\begin{gathered}
\boldsymbol{K}_{A}=\tilde{\boldsymbol{K}}_{A_{w w}}-\tilde{\boldsymbol{K}}_{A_{w \theta}} \tilde{\boldsymbol{K}}_{A_{\theta \theta}}^{-1} \tilde{\boldsymbol{K}}_{A_{\theta w}} \\
\boldsymbol{p}_{A}=\boldsymbol{f}_{A}-\tilde{\boldsymbol{K}}_{A_{w \theta}} \tilde{\boldsymbol{K}}_{A_{\theta \theta}}^{-1} \boldsymbol{m}_{A}
\end{gathered}
$$

The condensed stiffness matrix $\boldsymbol{K}_{A}$ is of size $(7 \times 7)$. This model shows that the stiffness of the array changes considerably with the angle $\alpha$, as observed in the experiments. The reason for this is that the structure is far more flexible, during the middle part of deployment, due to twisting of the panels, than it is when it is fully deployed or retracted. In these extreme configurations only bending occurs, a deformation mode where the panels exhibit a much stiffer behavior.

\section{Model of Suspension System}

The suspension system consists of the four independent suspension elements I, II, III, and IV. These are complex structures to model accurately, and, hence, it was decided to measure their stiffness experimentally. 


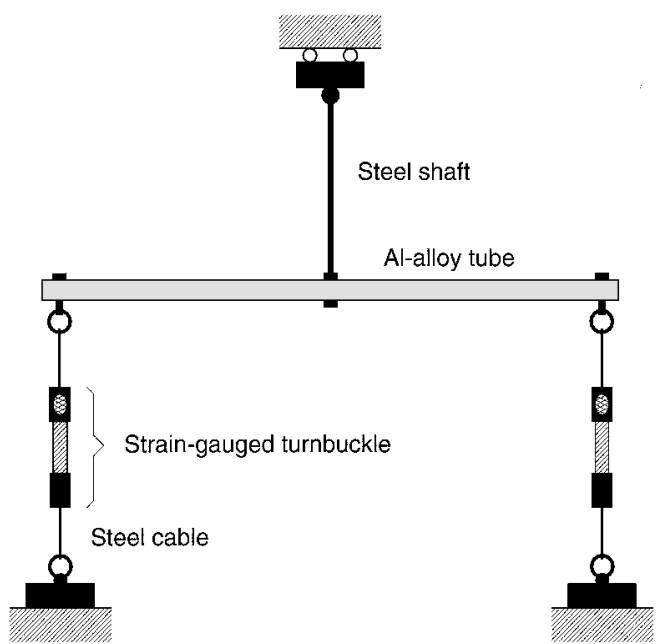

Fig. 3 Suspension element test rig.

To establish the relationship between the vertical displacements $\boldsymbol{w}_{S}$ and the associated forces $\boldsymbol{p}_{S}$ at the suspension points, the suspension elements were taken out of the assembly and set up in a separate test rig (Fig. 3).

For each suspension element a series of displacement-forcemeasurements were taken. The displacements were applied as length changes of the turnbucklesin the cables and measured with a Vernier caliper. The resulting strains in the suspensioncables were measured by the strain gauges.

The three double-point suspension elements II, III, and IV are designed identically and, thus, should have the same structural properties. Their stiffness matrices $\boldsymbol{K}_{S_{I I}}=\boldsymbol{K}_{S_{I I I}}=\boldsymbol{K}_{S_{I V}}$, relating $\boldsymbol{w}_{S}$ to $\boldsymbol{p}_{S}$, were calculated by fitting the experimental data with a set of global parameters determined by a least-squares solution. Determination of the stiffness $\boldsymbol{K}_{S_{I}}$ of the single-point suspension element I was done independently, using the same technique.

The $(7 \times 7)$ stiffness matrix $\boldsymbol{K}_{S}$ representing the complete suspension system is composed of the independent matrices $\boldsymbol{K}_{S_{I}}-\boldsymbol{K}_{S_{I V}}$ of the four suspension elements

$$
\boldsymbol{K}_{S} \boldsymbol{w}_{S}=\left[\begin{array}{llll}
\boldsymbol{K}_{S_{I}} & & & \\
& \boldsymbol{K}_{S_{I I}} & & \\
& & \boldsymbol{K}_{S_{I I I}} & \\
& & & \boldsymbol{K}_{S_{I V}}
\end{array}\right] \boldsymbol{w}_{S}=\boldsymbol{p}_{S}
$$

In contrast to the array structure, the stiffness of the suspension system does not depend on the deployment angle $\alpha$, and, thus, remains constant throughout deployment.

\section{Complete Model}

The stiffness matrix $\boldsymbol{K}_{C}$ of the complete structure formed by the solar array and suspension system relates the vertical displacement $\boldsymbol{w}_{C}$ to the applied loads $\boldsymbol{p}_{C}$ at the suspension points. It is derived by combining the stiffness expressions of the individual systems, Eqs. (3) and (6), by means of the compatibility condition

$$
\boldsymbol{w}_{A}=\boldsymbol{w}_{S}=\boldsymbol{w}_{C}
$$

and the equilibrium condition

$$
\boldsymbol{p}_{A}=\boldsymbol{p}_{C}-\boldsymbol{p}_{S}
$$

From these equations the stiffness relationship for the combined structure is

$$
\boldsymbol{K}_{C} \boldsymbol{w}_{C}=\left(\boldsymbol{K}_{A}+\boldsymbol{K}_{S}\right) \boldsymbol{w}_{C}=\boldsymbol{p}_{C}
$$

$\boldsymbol{K}_{C}$ has dimensions $(7 \times 7)$ and depends on the deployment angle $\alpha$.

No numbers for the stiffness matrices shall be given here, but note that in the fully deployed or retracted conditions the stiffness of the array structure is about three to four orders of magnitude higher than that of the suspension system, whereas during deployment the stiffnesses of the array and the suspension are comparable.

\section{Relationship Between Suspension Forces and Adjustments}

The suspension forces can be redistributed by adjusting the turnbuckles in the suspension system. A relationshipbetween the length changes $\boldsymbol{\delta}$ of the suspension cables and the change in suspension forces $\Delta s$ needs to be established. To do this, the computational models for the array and the suspension system need to be combined, taking into account the length changes $\delta$. The equilibrium condition (8) still applies, but no external loads need to be considered because there is no change in the external loads. Therefore, the forces acting on the two systems are due to the forces $s$ in the suspension cables only. Hence, the force changes are

$$
\Delta p_{A}=-\Delta p_{S}=\Delta s
$$

The displacementcondition has to be modified to include the length changes $\delta$ at the suspension cables and, therefore,

$$
\Delta \boldsymbol{w}_{A}=\Delta \boldsymbol{w}_{S}+\boldsymbol{\delta}
$$

where positive $\delta$ correspond to shortening of the suspension cables.

Equations (3), (6), (10), and (11) give

$$
\Delta \boldsymbol{w}_{A}=\left(\boldsymbol{K}_{A}+\boldsymbol{K}_{S}\right)^{-1} \boldsymbol{K}_{S} \boldsymbol{\delta}
$$

that, together with Eqs. (3) and (10), yields the desired $(\boldsymbol{\delta}, \Delta \boldsymbol{s})$ relationship

$$
\boldsymbol{R} \delta=\Delta s
$$

Here

$$
\boldsymbol{R}=\boldsymbol{K}_{A}\left(\boldsymbol{K}_{A}+\boldsymbol{K}_{S}\right)^{-1} \boldsymbol{K}_{S}=\boldsymbol{K}_{A} \boldsymbol{K}_{C}^{-1} \boldsymbol{K}_{S}
$$

which provides the essential tool for the manipulation and redistribution of the suspension forces.

The $(7 \times 7)$ matrix $\boldsymbol{R}$ is not a stiffness matrix in the usual sense because it relates internal forces and displacements, not externally applied loads and displacements.

\section{Variation of Suspension Forces During Deployment}

\section{Predicted and Measured Suspension Forces}

The combined system formed by the solar array and the suspension system is loaded by the self-weight of the array. The self-weight of the suspension system need not be considered because it is equilibrated directly within the system itself. The mass distribution of the array has been estimated from measurements and calculations. In the physical model $\sim 80 \%$ of the total mass is due to the hinges and the tip mass, and the remaining $\sim 20 \%$ is due to the panels (see Table 1).

Forces resulting from the masses concentrated at the hinges are applied as single loads $\boldsymbol{F}_{H}$. The uniformly distributed mass of the panels gives rise to appropriate equivalentnodal loads $\boldsymbol{F}_{P}, \boldsymbol{M}_{P_{x}}$, and $\boldsymbol{M}_{P_{y}}$ that depend on the actual deployment configuration. Hence, the load vector $\tilde{\boldsymbol{p}}_{C}$ is

$$
\tilde{\boldsymbol{p}}_{C}=\left(\begin{array}{c}
\boldsymbol{f}_{C} \\
\boldsymbol{m}_{C}
\end{array}\right)=\left[\begin{array}{c}
\boldsymbol{F}_{H}+\boldsymbol{F}_{P} \\
\boldsymbol{M}_{P_{x}} \\
\boldsymbol{M}_{P_{y}}
\end{array}\right]
$$

In analogy with the matrix condensation of the array stiffness matrix $\tilde{\boldsymbol{K}}_{A}$, the load vector $\tilde{\boldsymbol{f}}_{C}$ can be reduced from 19 to 7 elements to match the stiffness matrix $\boldsymbol{K}_{C}$ of the combined system [Eq. (5)], and the condensed load vector $\boldsymbol{p}_{C}$ is then given by

$$
\boldsymbol{p}_{C}=\boldsymbol{f}_{C}-\tilde{\boldsymbol{K}}_{A_{w \theta}}\left(\tilde{\boldsymbol{K}}_{A_{\theta \theta}}\right)^{-1} \boldsymbol{m}_{C}
$$

This loading is applied on the computationalmodel of the combined system [Eq. (9)] to determine the resulting displacements at the suspension points.

Then, by using the stiffness relationshipfor the suspension system [Eq. (6)], the internal forces in the suspension cables can be recovered. Figure 4 shows the forces in the suspension cables throughout deployment. 


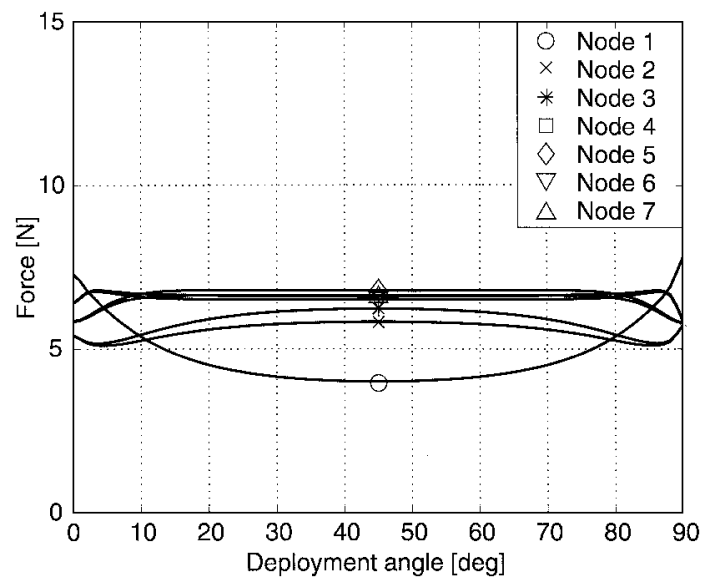

Fig. 4 Predicted variation of suspension forces assuming perfect alignment.

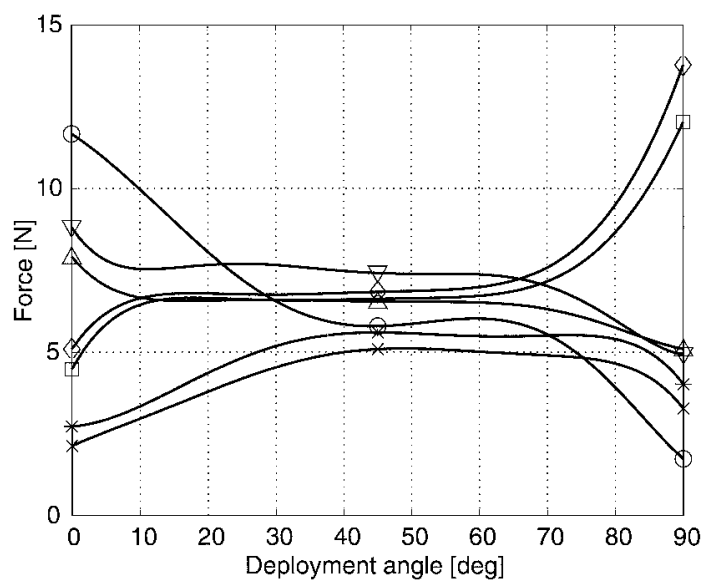

a) Experiment a

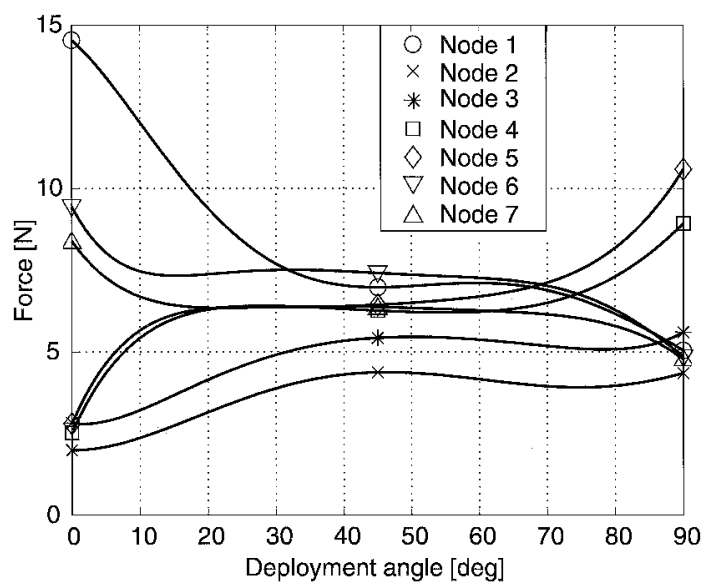

b) Experiment b

Fig. 5 Measured variation of suspension forces.

The computational model represents an idealized structure with no misalignment or imperfections where the hinges are exactly vertical, the suspension elements are identical, and the rails supporting the bearings of the suspension system are straight and horizontally level. In practice, though, all of these imperfections occur to an unknown extent and, thus, it might be expected that the measured suspension forces will not even resemble the suspension forces resulting from a perfect computational model. Also, the initial length adjustments of the suspension cables influence significantly the variation of the suspension forces during deployment. Figure 5 shows only two examples of the many suspensionforce variations that were measured.

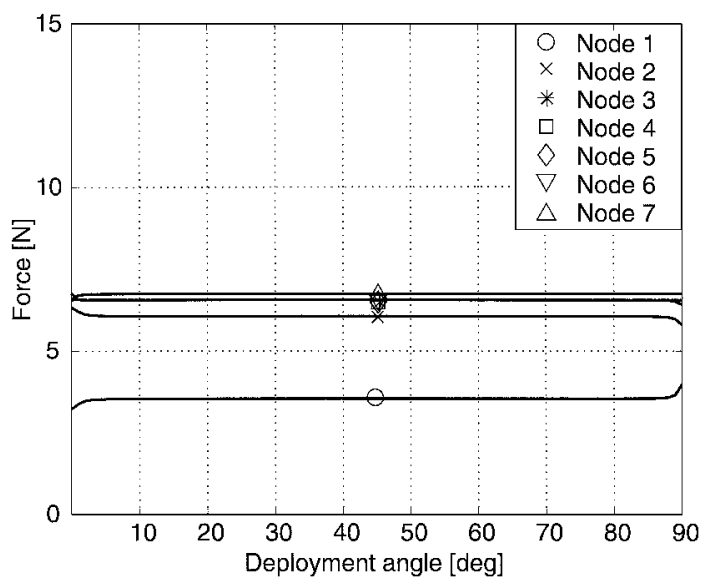

Fig. 6 Required variation of suspension forces.

However, we can use the relationship for the redistribution of the suspension forces [Eq. (13)] to change the current force distribution by computing the adjustments that will best simulate a weightless environment.

\section{Required Suspension Forces}

During deployment the forces carried by the hinges should be as small as possible if the suspension system is to accurately replicate the behavior of the structure in space. This requires that the deformation of the array be minimized and, hence, that the displacement of all suspension points be a pure translation.

The forces acting on the suspension points of the array are the loads $\boldsymbol{p}_{C}$ due to self-weight, given by Eq. (16), and the suspension cable forces $s$ :

$$
\boldsymbol{K}_{A} \boldsymbol{w}_{A}=\boldsymbol{p}_{C}+\boldsymbol{s}
$$

The stiffness matrix $\boldsymbol{K}_{A}$ of the array without the suspension system is singular, because the structure has a degree of kinematic freedom in the vertical direction. Therefore, its displacements need to be considered relative to the displacement $w_{1}$ of suspension point one

$$
\boldsymbol{K}_{A}^{*}\left(\boldsymbol{w}_{A}-\boldsymbol{w}_{A_{1}}\right)=\boldsymbol{p}_{C}+\boldsymbol{s}
$$

where $\boldsymbol{K}_{A}^{*}$ is a $(7 \times 6)$ reduced version of $\boldsymbol{K}_{A}$.

Pure translation of the array occurs for the relative displacements $\left(\boldsymbol{w}_{A}-\boldsymbol{w}_{A_{1}}\right)=0$. To achieve this, no resulting forces are to act at the suspension points, which in turn implies that at any stage during deployment the suspension forces have to exactly equilibrate the self-weight loads at every suspension point. Hence, the required suspension forces $s_{R}$ for proper gravity compensation are

$$
\boldsymbol{s}_{R}=-\boldsymbol{p}_{C}
$$

whose dependence on the deployment angle $\alpha$ is plotted in Fig. 6 .

As could be expected, the required distribution of suspension forces is almost constant because the main part of the loading is applied as concentrated loads that are supported right at the suspension points for any configuration of the array. However, there would be a more significant variation if the mass of the system were more uniformly distributed. Indeed, this would be the case for an array structure of larger scale, where the mass of the hinges would be much smaller than the mass of the panels.

The suspension force at node 1 is smaller than that at the other nodes because one of the panels connected to this hinge is only half as big as the other panels, and, hence, its mass is much lower.

\section{Adjustment of Suspension System}

Experiments were carried out to verify the computational models and also to check the accuracy of the method for redistributionof the suspension forces by adjusting the length of the suspension cables.

In the experiments, deployment and retraction were set to last $90 \mathrm{~s}$. Every second, the strain gauge readings were recorded by a data logger, so that the forces in the suspension cables are available 
at intervals of $1 \mathrm{deg}$ of the deployment angle $\alpha$. The preparation for these experiments included resetting the strain gauges to zero by unloading the suspension cables one after the other, so that the sum of the strain gauge readings always corresponded to the overall supported weight of the array. Measurements were taken for both deployment and retraction and then averaged at corresponding deployment angles. Noise was removed by smoothing out the data using polynomial fitting.

Comparing the measured suspension force distributions $\boldsymbol{s}_{M}$ (Fig. 5) to the required suspension forces $s_{R}$ (Fig. 6) the necessary changes in suspension forces are determined by

$$
\Delta s=s_{M}-s_{R}
$$

Then, the necessary length adjustments of all seven suspension cables were calculated with Eq. (13) and are plotted in Fig. 7.

When comparing Figs. 5 and 6, note that their discrepancy is relatively big at either end of deployment, whereas the agreement is much better in the range $\alpha=40-70 \mathrm{deg}$. This is due to the reduction in stiffness of the array structure during the middle part of deployment, as described before. Therefore, vertical deviations of the suspension points from their required positions result in much larger reaction forces than in the end configurations. In intermediate configurations, the array can yield much more easily to the restraints imposed by the suspension system. For the same reason, the length adjustments there need to be impractically large to achieve only minor force corrections, in contrast to the end configurations where the deviation of the actual from the required forces is much bigger but can be corrected by much smaller adjustments.

Our experimental setup allows adjustment in only one particular configuration because the length changes at the suspension wires have to be imposed manually. Therefore, either the fully retracted or the fully deployed configuration is chosen, where large force discrepancies can be corrected by small length adjustments.

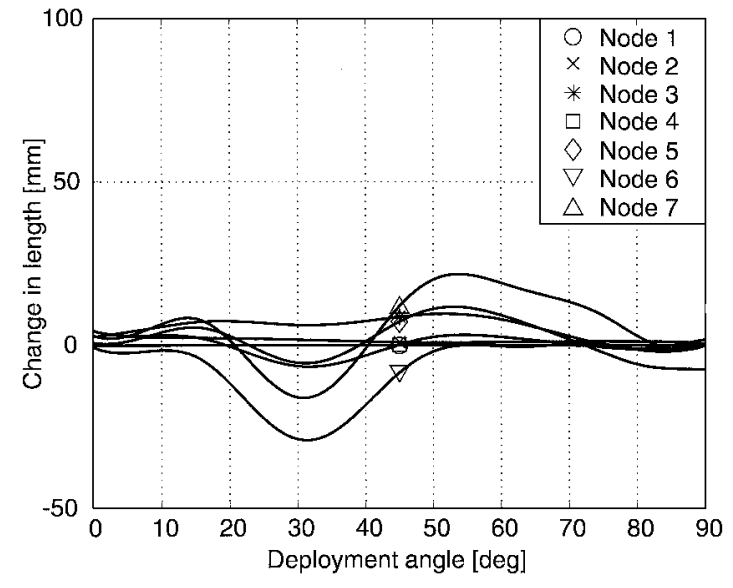

a) Experiment a

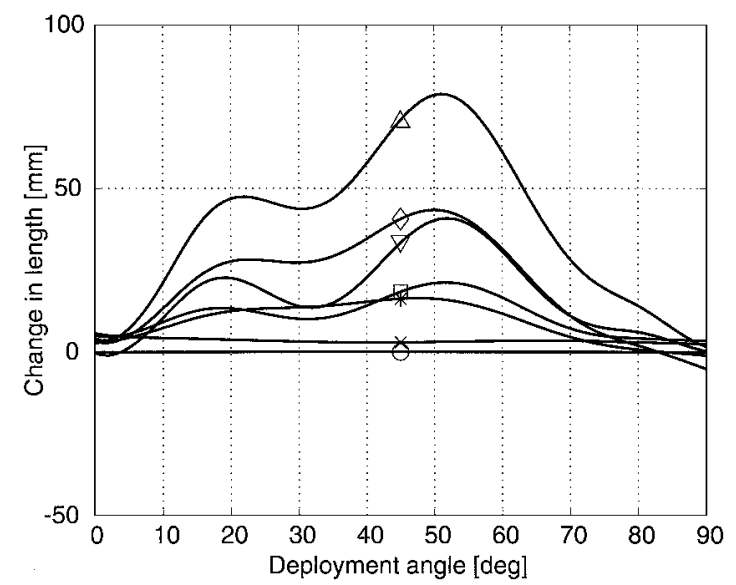

b) Experiment b

Fig. 7 Required suspension cable adjustments.
Because of the array structure itself having one degree of freedom in the vertical direction, identical length changes at all suspension cables would not affect the force distribution. The necessary length changes are, therefore, calculated with respect to suspension point 1 . This implies reducing matrix $\boldsymbol{R}$ in Eq. (13) from size $(7 \times 7)$ to $(7 \times 6)$ and then solving for the length changes in a least-squares sense. The length changes computed thus are then translated vertically to minimize the amount of manual adjustment at each point and to stay within the range of the turnbuckles.

The suspension forces $s_{P}$ that are predicted by the computational model when the suspension cables are adjusted a) in the fully deployed and b) in the fully retracted configuration, that is,

$$
\boldsymbol{S}_{P}=\boldsymbol{s}_{M}+\left.\Delta \boldsymbol{S}\right|_{\begin{array}{l}
(a) \alpha=0 \mathrm{deg} \\
(b) \alpha=90 \mathrm{deg}
\end{array}}=\boldsymbol{s}_{M}+\left.\boldsymbol{R} \boldsymbol{\delta}\right|_{\begin{array}{l}
(a) \alpha=0 \mathrm{deg} \\
(b) \alpha=90 \mathrm{deg}
\end{array}}
$$

are shown in Fig. 8. As a comparison, Fig. 9 shows the forces that were measured on our model after actually making the required adjustments.

The theoretical predictions and measurements are in good agreement throughoutdeployment, thus showing that the model correctly describes the behavior of this shape-varying structure and its interaction with the gravity compensation system. In the fully deployed and retracted configurations, for which the adjustments were made, the agreement of both experiment and theoretical predictions with the required suspensionforce distributionis particularly satisfactory The adjustment has also improved the suspension force distribution during the middle part of the deployment, but not at the respective other end.

\section{Discussion}

For every particular deployment configuration, the system consisting of array and suspension exhibits different behavior and properties, demanding varying adjustment throughoutdeployment. Also,

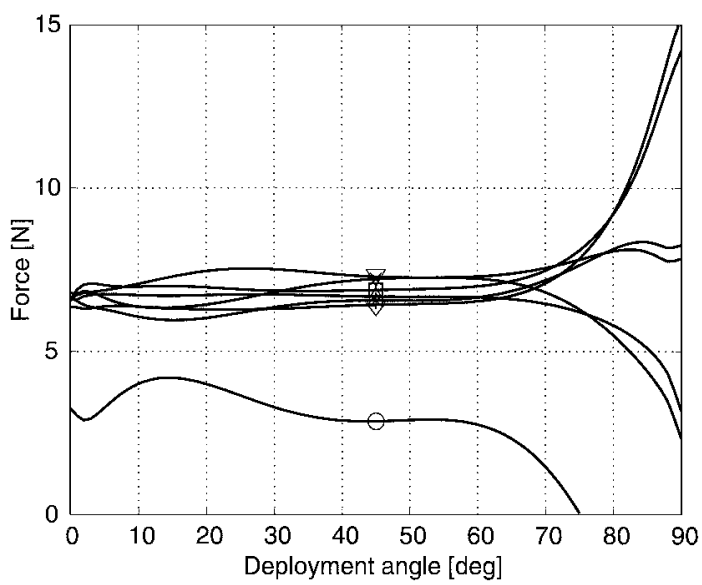

a) Experiment a

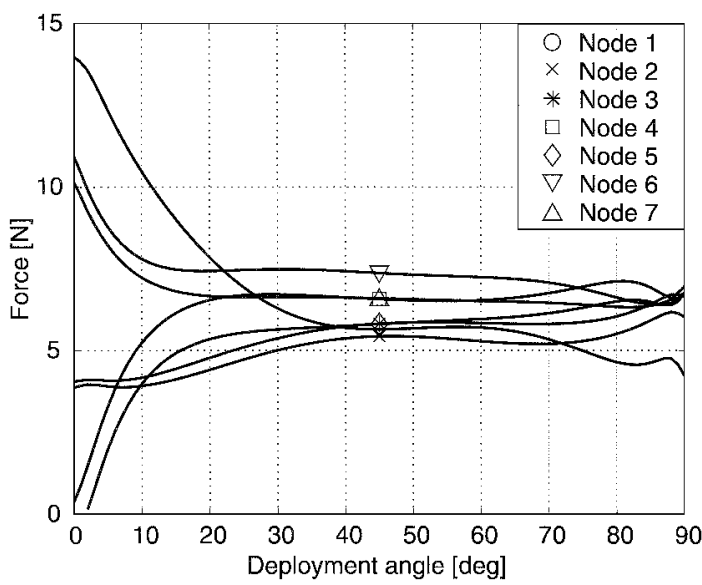

b) Experiment b

Fig. 8 Predicted variation of suspension forces after adjustment. 


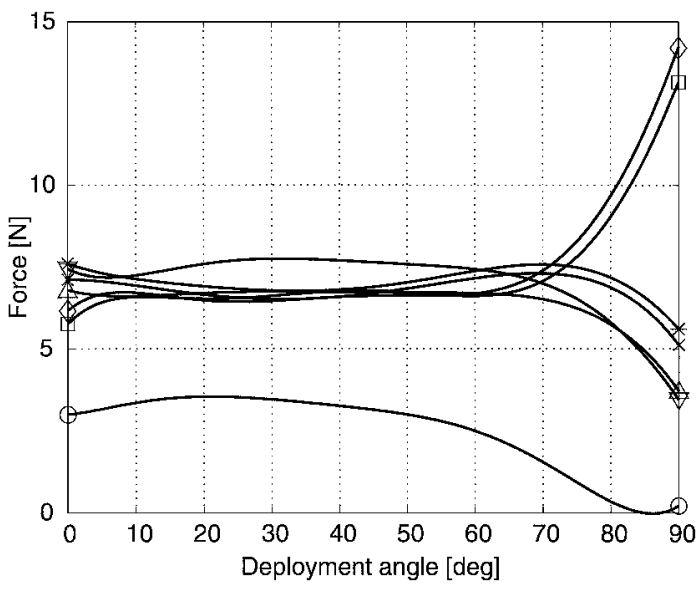

a) Experiment a

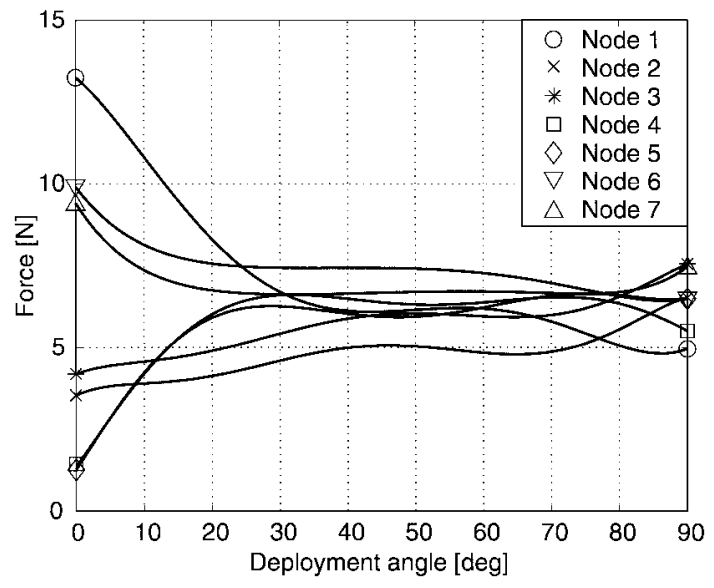

b) Experiment b

Fig. 9 Measured variation of suspension forces after adjustment.

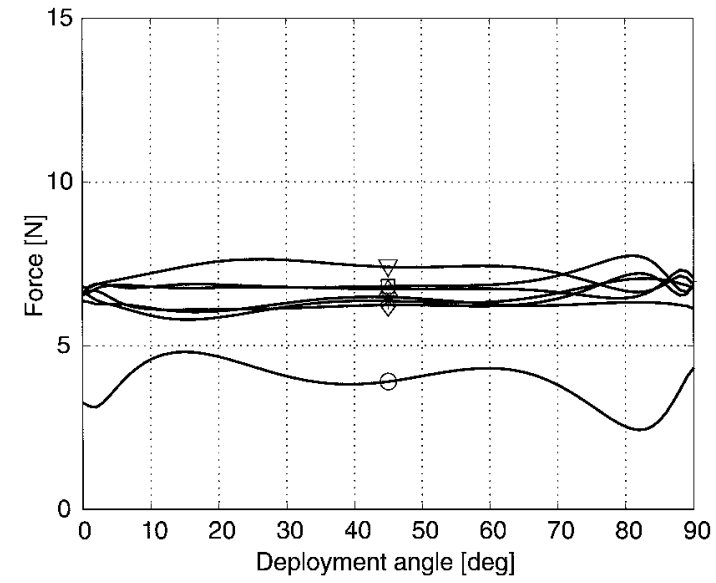

a) Experiment a

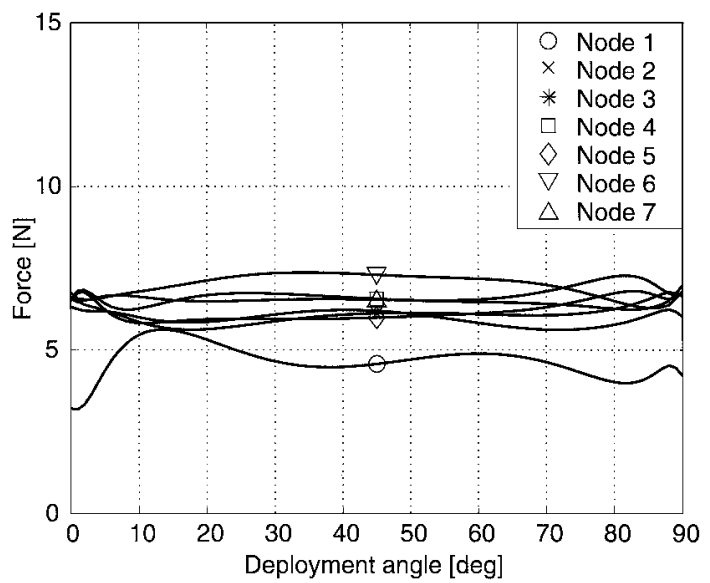

b) Experiment b

Fig. 10 Predicted variation of suspension forces after interpolated adjustment

initial imperfections and misalignment of the hinges and panels, end support, supporting rails, and the support framework affect the necessary adjustments at any deployment angle $\alpha$. This becomes most apparent in the two end configurations, where the structure is relatively stiff. Here, improving the force distribution at one end has the effect of making it worse at the other end.

Figure 10 shows the theoretical predictions for the suspension force distribution that could be achieved with a simple open-loop, active suspension system that applies the required length adjustments both in the fully deployed and fully retracted configurations and, in between these configurations, applies linearly interpolated adjustments. Even such a simple adjustment achieves suspension forces that are remarkably close to the required distribution. In practice, though, this type of variable adjustment cannot yet be tested on the existing experimental setup. Similar good agreement between computational predictions and experimental data, however, can be expected, as exhibited in the cases where adjustmentis only possible at either end of deployment.

\section{Conclusions}

In conclusion, this study has established a methodology for modeling the interactionbetween deployable structures and gravity compensation systems. It has been shown that the effects of adjustments to the suspension system can be accurately predicted and that some improvements in the distribution of forces applied by the system to the structure can be achieved by means of a single adjustment of the suspension system. However, to fully optimize the performance of the suspension system, active adjustment techniques will be required, allowing for variable adjustment while the structure moves. An even more advanced, closed-loop control algorithm may be needed to simulate zero gravity with respect to the dynamic behavior of the structure. Work on these topics has begun.

\section{Acknowledgments}

This work was carried out as part of a European Community training project funded through a Marie Curie Fellowship. The authors thankfully acknowledge financial support from Pembroke College, Cambridge, for A. Fischer and from the Royal Academy of Engineering for S. Pellegrino.

\section{References}

${ }^{1}$ Chung, D. T., and Young, L. E., "Zero Gravity Testing of Flexible Solar Arrays," NASA CP-2181, May 1981.

${ }^{2}$ Woodard, S. E., and Housner, J. M., "Nonlinear Behavior of a Passive Zero-Spring-Rate Suspension System," Journal of Guidance, Control, and Dynamics, Vol. 14, No. 1, 1989, pp. 84-89.

${ }^{3}$ Kienholz, D. A., Crawley, E. F., and Harvey, T. J., "Very Low Frequency Suspension Systems for Dynamic Testing," Proceedings of the 13th Structures, Structural Dynamics, and Materials Conference, AIAA, Washington, DC, 1989, pp. 327-336.

${ }^{4}$ Jagannathan, S., and Fenn, R. C., "Low-Cost Active Anti-Gravity Suspension System," Proceeding s of the American Control Conference, Seattle, WA, 1995, pp. 3606-3611.

${ }^{5}$ Cooley, V. M., and Giunta, A. A., "Laboratory Evaluation of Two Advanced Suspension Devices for Ground Vibration Testing of Large Space Structures," Proceedings of the 33rd Structures, Structural Mechanics, and Materials Conference, AIAA, Washington, DC, 1992, pp. 1700-1710.

${ }^{6}$ Straube, T. M., and Peterson, L. D., "A Large Motion Suspension System for Simulation of Orbital Deployment," Proceedings of the 35th Structures, 
Structural Mechanics, and Materials Conference, AIAA, Washington, DC, 1994,pp. 1959-1969.

${ }^{7}$ Yang, L. F., Tzeng, R. C., and Kuo, C. P., "Adaptive Control of a ZeroStiffness Suspension System," Journal of Guidance, Control, and Dynamics, Vol. 19, No. 4, 1996, pp. 794-800.

${ }^{8}$ Brown, H. B., and Dolan, J. M., "A Novel Gravity Compensation System for Space Robots," Proceedings of the ASCE Speciality Conference on Robotics for Challenging Environments, American Society of Civil Engineers, Albuquerque, NM, 1994, pp. 250-258.

${ }^{9}$ White, G. C., and Xu, Y., "An Active Vertical-Direction Gravity Compensation System," IEEE Transactions on Instrumentation and Measurement, Vol. 43, No. 6, 1994, pp. 786-792.

${ }^{10}$ Hasselman, T. K., and Quartararo, R., "A Suspension System for Large Amplitude Dynamic Testing in a Simulated Weightless Environment," Society of Photo-Optical Instrumentation Engineers/American Society of Mechanical Engineers/Society of Experimental Mechanics North American
Conf. on Smart Structures and Materials, Albuquerque, NM, Feb. 1993.

${ }^{11}$ Zwanenburg, R., "The Qualification Test Programme of the ENVISAT Solar Array Mechanism," Proceedings of the Sixth European Space Mechanisms and Tribology Symposium, 1995, pp. 65-72; ESA, SP-374, Zurich, Aug. 1995.

${ }^{12}$ Sato, Y., Ejiri, A., Iida, Y., Kanda, S., Maruyame, T., Uchiyama, T., and Fujii, H., "Micro- $g$ Emulation System Using Constant-Tension Suspension for a Space Manipulator," Proceedings of the IEEE International Conference on Robotics and Automation, Inst. of Electrical and Electronics Engineers, 1991,pp. 1893-1900.

${ }^{13}$ Kumar, P., and Pellegrino, S., "Deployment and Retraction of a CableDriven Rigid Panel Solar Array,” Journal of Spacecraft and Rockets, Vol. 33, No. 6, 1996, pp. 836-842.

R. B. Malla Associate Editor 